\title{
Stress administered prior to encoding impairs neutral but enhances emotional long-term episodic memories
}

\author{
Jessica D. Payne, ${ }^{1,3,4}$ Eric D. Jackson, ${ }^{2}$ Siobhan Hoscheidt, ${ }^{2}$ Lee Ryan, ${ }^{2}$ W. Jake Jacobs, ${ }^{2}$ \\ and Lynn $\mathrm{Nadel}^{2,4}$ \\ ${ }^{1}$ Harvard University, Department of Psychology, William James Hall, Cambridge, Massachusetts 02138, USA; ${ }^{2}$ University of \\ Arizona, Department of Psychology, Tucson, Arizona 85721, USA; ${ }^{3}$ Beth Israel Deaconess Medical Center, Harvard Medical \\ School, Department of Psychiatry, Boston, Massachusetts 02215, USA
}

\begin{abstract}
Stressful events frequently comprise both neutral and emotionally arousing information, yet the impact of stress on emotional and neutral events is still not fully understood. The hippocampus and frontal cortex have dense concentrations of receptors for stress hormones, such as cortisol, which at high levels can impair performance on hippocampally dependent memory tasks. Yet, the same stress hormones can facilitate memory for emotional information, which involves interactions between the hippocampus and amygdala. Here, we induced psychosocial stress prior to encoding and examined its long-term effects on memory for emotional and neutral episodes. The stress manipulation disrupted long-term memory for a neutral episode, but facilitated long-term memory for an equivalent emotional episode compared with a control condition. The stress manipulation also increased salivary cortisol, catecholamines as indicated by the presence of $\boldsymbol{\alpha}$-amylase, heart rate, and subjectively reported stress. Stressed subjects reported more false memories than nonstressed control subjects, and these false memories correlated positively with cortisol levels, providing evidence for a relationship between stress and false memory formation. Our results demonstrate that stress, when administered prior to encoding, produces different patterns of long-term remembering for neutral and emotional episodes. These differences likely emerge from differential actions of stress hormones on memory-relevant regions of the brain.
\end{abstract}

Stress profoundly influences memory in humans and other species (Kim and Diamond 2002; Roozendaal 2002). This effect is in part due to activation of the hypothalamic-pituitary-adrenal (HPA) axis, which elicits a cascade of stress hormones and culminates in the release of glucocorticoids (GCs) from the adrenal cortex. Many of the brain regions important for memory (hippocampus, prefrontal cortex, amygdala) have dense concentrations of GC receptors (Lupien and Lepage 2001), and the function of these regions can be influenced by elevated stress hormones (de Quervain et al. 2003).

Stress or GC treatment can either impair (de Quervain et al. 2000), or enhance (Buchanan and Lovallo 2001; Cahill et al. 2003; Putman et al. 2004) memory performance, depending on several modulatory factors. One such factor is memory stage (i.e., acquisition/encoding, consolidation, retrieval). Glucocorticoids, interacting with adrenergic activation in the basolateral amygdala and the hippocampus, appear to impair delayed memory retrieval, but enhance memory consolidation (Kuhlmann et al. 2005a,b; Buchanan et al. 2006; but, see Diamond et al. 2006 for an example of stress-induced consolidation impairment).

The timing of the stress manipulation determines which stage of memory will be affected by cortisol elevations. For example, consolidation is tested by administering stress or cortisol treatment immediately after training, and then testing retrieval after a long delay (e.g., Cahill et al. 2003), whereas retrieval is targeted by allowing the memory to be acquired and consolidated normally, and then administering stress before retrieval

\footnotetext{
${ }^{4}$ Corresponding authors.

E-mail jdpayne@bidmc.harvard.edu; fax (617) 667-8498.

E-mail nadel@u.arizona.edu; fax (520) 621-9306.

Article is online at http://www.learnmem.org/cgi/doi/10.1101/lm.743507.
}

(e.g., Kuhlmann et al. 2005b) (see de Quervain et al. 2000 for a design targeting all three memory stages).

Although we are beginning to understand the impact of stress on memory consolidation and retrieval in humans, it remains unclear how stress initiated prior to encoding affects later remembering. Because cortisol does not return to baseline levels for at least 90 min following a stressor (Dickerson and Kemeny 2004), both encoding and early consolidation processes are affected in such designs. Nonetheless, examining the impact of pre-training stress on long-term memory retention is important because it reflects how stress often operates in the real world. Stress can occur prior to or during encoding of an event that one may need to remember sometime later (e.g., in the case of eyewitness testimony).

Several studies have shown an impact of pre-training stress on memory assessed immediately or after very brief delays (Wolf et al. 2001; Payne et al. 2002; Jelicic et al. 2004). However, these designs could not distinguish the effects of stress on encoding/ consolidation from those of stress on retrieval, because cortisol remained elevated during all three memory stages. Because our focus was specifically on encoding and consolidation processes, we exposed subjects to a stressor prior to encoding, and then tested memory 1 wk later, after cortisol levels had returned to normal (Kirschbaum et al. 1996; see also Buchanan and Lovallo 2001).

Another factor modulating the impact of stress on memory is emotional arousal. Stress often impairs memory for neutral materials (de Quervain et al. 2000; Payne et al. 2002; Tops et al. 2003), while it enhances memory for emotionally arousing materials (Buchanan and Lovallo 2001; Cahill et al. 2003). This divergent pattern may be due in part to the action of GCs on the 
different brain regions involved in memory: The function of the hippocampus and regions of the prefrontal cortex may be impaired, while amygdala function may be enhanced by their elevation (Nadel and Jacobs 1998; Vyas et al. 2002).

In a previous study (Payne et al. 2006), we showed that memories for emotional and neutral information contained within a single episode were differentially affected by pretraining stress (neutral information was disrupted while emotional information was preserved relative to a no-stress control group). However, we were unable to confirm (1) that emotional information was in fact enhanced by stress exposure (i.e., we failed to find the crossover where stress both disrupted memory for neutral information and enhanced memory for emotional information), and (2) that the observed effects were due to measured changes in stress responsivity.

In the present study, we initiated stress before encoding, but this time we aimed (1) to examine its impact on the long-term retention of separate emotional and neutral episodes, and (2) to determine whether changes in memory performance would correlate with measures of stress responsivity (cortisol, catecholamines, heart rate). We predicted that stress would enhance memory for emotionally arousing negative material but disrupt memory for emotionally neutral material, and that both of these effects would be driven by elevations in stress hormones.

\section{Results}

\section{Cortisol levels}

As expected, the Trier Social Stress Test (TSST) significantly elevated cortisol levels (Fig. 1A). A repeated-measures ANOVA with sex and stress group as between-subject factors and assessment time (pre-TSST, post-TSST, 30-min post-TSST, 1-wk return) as the within-subject factor, revealed a significant main effect of stress group $\left(F_{[1,62]}=5.9, P<0.02\right)$ and a significant stress group by assessment time interaction $\left(F_{[3,186]}=8.0, P<0.0001\right)$. To examine the difference between stress and control groups at each of the four time points, we applied a Bonferroni correction to avoid Type 1 error. We adjusted the $\alpha$ level from 0.05 to 0.05 divided by four, or 0.0125 , and considered values of $P \leq 0.01$ to be significant. These Bonferroni corrected $t$-tests indicated that stress exposure significantly elevated cortisol levels immediately after the stressor and 30-min after the stressor $(t(74)=3.2, P=0.002$; $t(74)=3.1, P=0.003$, respectively). The stress group did not differ from the control group, however, either at baseline or at the return visit. No significant sex differences were detected.

\section{Catecholamines}

Analysis of catecholamines, as indexed by salivary $\alpha$-amylase, used an identical repeated-measures ANOVA with sex and stress group as between-subject factors and assessment time (two levels: pre-manipulation and post-manipulation) as the within-subject factor. The ANOVA revealed a significant stress group by assessment time interaction $\left(F_{[1,72]}=19.2, P<0.0001\right)$. As expected, Bonferroni corrected $t$-tests ( $\alpha$ level adjusted to $P=0.02$ ) revealed that participants in the stress group had significantly higher catecholamine levels following TSST exposure (Time 2) than they did before exposure (Time 1) $(M s=94.9 \mathrm{U} / \mathrm{mL}$ vs. $79.0 \mathrm{U} / \mathrm{mL}$; $t(35)=3.5, P=0.003)$. Unexpectedly, however, participants in the control group had significantly lower catecholamine levels at Time 2 than at Time $1(\mathrm{Ms}=92.2 \mathrm{U} / \mathrm{mL}$ vs. $81.6 \mathrm{U} / \mathrm{mL}$; $t(39)=2.6, P=0.01$ ), which may have resulted from the relief of not being assigned to the public-speaking condition. Importantly, the stress and control groups did not differ in their $\alpha$ amylase values at the Time 1 baseline $(M s=79.0 \mathrm{U} / \mathrm{mL}$ vs. 92.2 $\mathrm{U} / \mathrm{mL}, P=0.17)$. Again, no significant sex differences were detected.

\section{Subjective stress}

A repeated-measures ANOVA, again with sex and stress group as between-subject factors and assessment time as the withinsubject factor, revealed a significant main effect of stress group $\left(F_{[1,70]}=51, P<0.0001\right)$ and a significant stress group by assess-
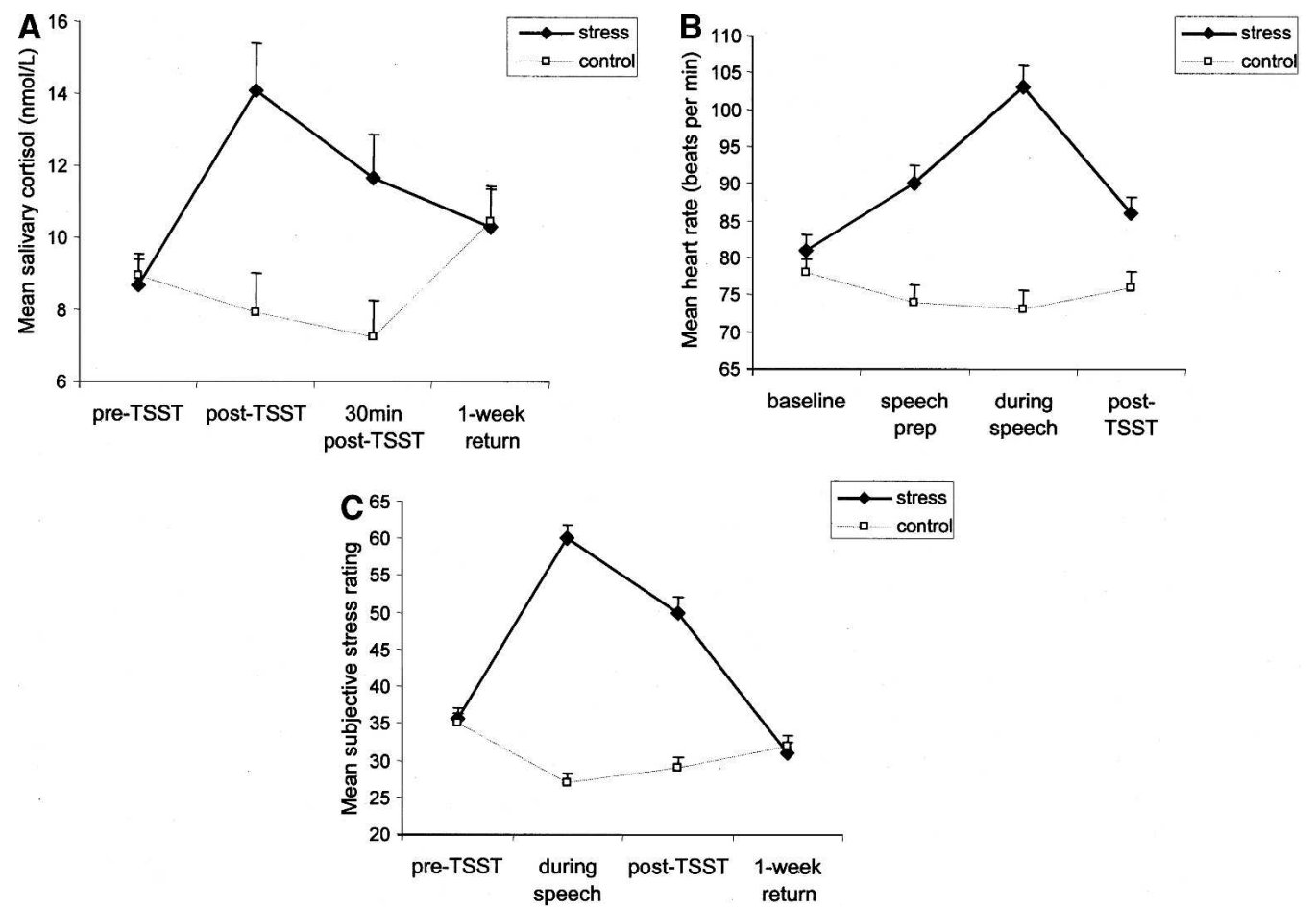

Figure 1. Mean salivary cortisol, heart rate, and subjective stress ratings in the Stress and Control groups. 


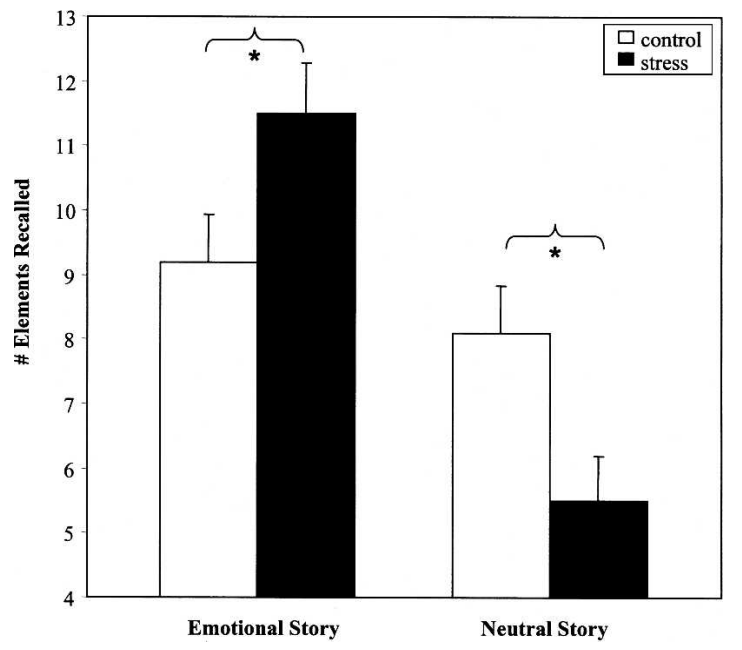

Figure 2. The impact of stress on recall memory. Stress resulted in recall of more elements from the emotional story, but fewer elements from the neutral story, compared with the control condition.

ment time interaction $\left(F_{[3,210]}=95.2, P<0.0001\right)$ (Fig. 1C). Bonferroni corrected $t$-tests ( $\alpha$ level adjusted to $P=0.0125$ ) indicated that exposure to the stressor elevated self-reported anxiety levels immediately after $(t(74)=8.2, P<0.0001)$, and during (i.e., "how did you feel during the stressor") $(t(74)=14.0, P<0.0001)$ the stressor. Stressed subjects did not differ from controls, however, at baseline or the return visit $(t(74)=0.37, P=0.71 ; t(74)=0.48$, $P=0.63)$.

There was also a significant three-way interaction among sex, stress group, and assessment times $\left(F_{[3,210]}=4.3, P<0.01\right)$. Post-hoc $t$-tests indicated that both males and females exhibited the same pattern of stress effects as above; however, females reported significantly higher levels of self-reported anxiety than males during and after the stressor $(t(74)=2.4, P<0.02$; $t(74)=2.1, P<0.05)$.

\section{Heart rate}

A repeated-measures ANOVA with sex and stress groups as between-subject factors and assessment time (baseline, during the speech preparation, during the speech, and post-TSST) as the within-subject factor, revealed a significant main effect of time $\left(F_{[1,72]}=5.9, P<0.0001\right)$ and an interaction of time and stress group $\left(F_{[3,186]}=59, P<0.0001\right)$. Bonferroni corrected $t$-tests $(\alpha$ level adjusted to $P=0.0125$ ) indicated that exposure to the TSST significantly elevated heart rate over that of the control group (baseline: $t(64)=1.6$, ns; speech preparation: $t(64)=4.8$, $P<0.001$; speech task: $t(64)=7.8, P<0.001$; post-TSST: $t(64)=3.6$, $P<0.001$ ) (Fig. 1B).

\section{Memory performance}

Free recall

A $2 \times 2 \times 2 \times 3$ mixed ANOVA comparing group (stress, control), slide show (emotional, neutral), and sex (male, female), with phase of slide show (phases 1-3) as the within subjects factor, detected significant main effects of story type $\left(F_{[1,68]}=13.2\right.$, $P<0.001)$, and phase $\left(F_{[2,136]}=32.4, P<0.0001\right)$, demonstrating that $1 \mathrm{wk}$ after stress exposure, the emotional story was better recalled than the neutral story overall, and that phase 2 (the phase containing the emotional slides) was better recalled than phases 1 and 3. Importantly, there was a significant interaction between stress group and story type $\left(F_{[1,68]}=5.1, P<0.03\right)$. Independent samples $t$-tests indicated that stress significantly in- creased the recall of the emotional story $(t(37)=2.1, P<0.05)$, and significantly decreased recall of the neutral story, $(t(35)=2.0, P<0.05)$ compared with controls, while the controls showed similar recall of both stories (Fig. 2).

\section{Cued recall}

The pattern of results for the cued-recall data was generally the same as the free recall data. The critical story type $\times$ stress group interaction emerged, $F_{(1,68)}=8.2, P<0.01$, showing that stressed subjects recalled more of the slides from the emotional story a week later $(58 \%)$, but fewer of the slides from the neutral story (36\%), compared with the control group ( $44 \%$ and $42 \%$, respectively). However, while post hoc $t$-tests indicated that stress significantly increased recall of slides from the emotional story $(t(37)=2.4, P=0.02)$, stress only tended to decrease recall of slides from the neutral story $(t(35)=1.3, P=0.14)$.

\section{Recognition memory}

We next analyzed recognition memory scores with a $2 \times 2 \times 2$ between subjects ANOVA, comparing stress group (stress, control), story type (emotional, neutral), and sex (male, female). We again found the critical story type $\times$ group interaction, $F_{[1,67]}=9.6, P<0.01$. Independent samples $t$-tests confirmed that stressed subjects' recognition memory was both significantly impaired for the neutral slide show $(t(36)=5.1, P<0.05)$ and significantly enhanced for the emotional slide show $(t(36)=4.5$, $P<0.05)$, relative to control subjects, who showed similar scores on both stories (Fig. 3).

\section{False memory}

False slide reports were analyzed using a similar 2 (stress group) $\times 2$ (story type) $\times 2($ sex $) \times 3$ (phase) mixed ANOVA. A significant stress group $\times$ story type interaction, $F_{(1,68)}=4.0$, $P<0.05$ demonstrated an additional memory impairment in the stressed subjects (Fig. 4). Follow-up t-tests revealed significantly more falsely reported neutral slides in the stress group than in the control group $(t(36)=4.7, P<0.05)$. There was no difference between groups for falsely reported emotional slides $(P=0.21)$. In addition, a positive correlation emerged between false memories and cortisol levels $\left(\mathrm{r}_{(76)}=0.23, P<0.05\right)$-an effect which became even stronger when we analyzed cortisol responders separately (those secreting $12 \mathrm{nmoml} / \mathrm{L}$ or more in response to the TSST, $\left[\mathrm{r}_{(15)}=0.57, P<0.03\right.$, Fig. 5]) (Elzinga and Roelofs 2005; see

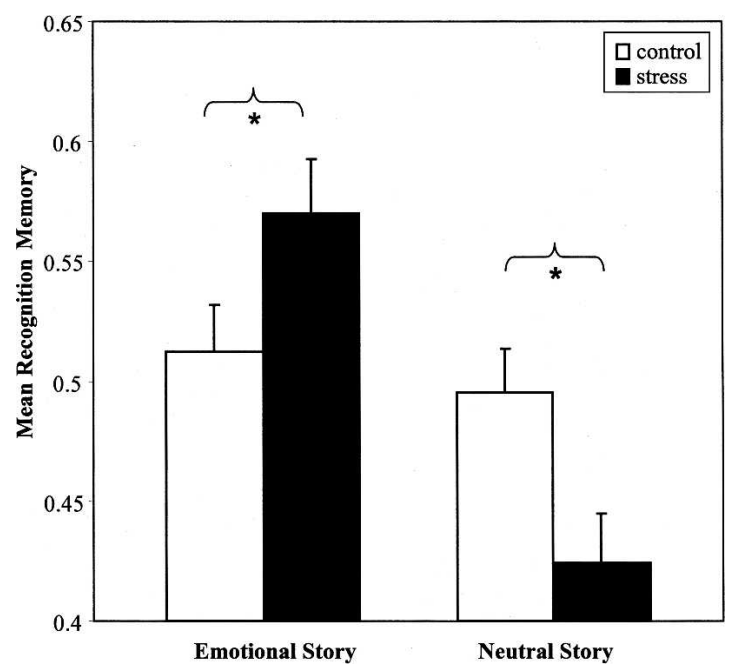

Figure 3. The impact of stress on recognition memory. Stress resulted in better recognition of the emotional story, but poorer recognition of the neutral story, compared with the control condition. 
Buchanan et al. 2006). The correlation between cortisol levels and false memory suggests that the stress group's memory deficit may be driven (at least in part) by elevated cortisol. However, this result should be interpreted with caution as the $\mathrm{N}$ is fairly small, and it was the only significant correlation to emerge between cortisol and the memory measures. Significant correlations did not emerge between the memory measures and $\alpha$-amylase or heart rate.

Sex was included in the above analyses because it is an important factor determining the impact of stress on memory (Wolf et al. 2001) Surprisingly, however, sex differences did not emerge in any of the memory analyses.

\section{Discussion}

As expected, exposure to a psychosocial stressor (the TSST) produced robust elevations in both cortisol and catecholamines. These effects, which were elicited shortly before encoding, had a marked impact on memory performance 1-wk later. Memory for emotionally arousing material was enhanced by the pre-training stress exposure, while memory for closely matched neutral material was impaired. These effects are quite different from those found when stress is administered before retrieval.

Stress administered before retrieval generally produces memory impairment, regardless of stimulus emotionality. Indeed, Kuhlmann et al. $(2005 a, b)$ have demonstrated that, under certain conditions, negative arousing material suffers more than neutral material. This is a fascinating finding because of the many real-world cases where stress accompanies memory retrieval (e.g., taking an exam). However, there are also important cases where stress accompanies memory encoding (e.g., rape, combat, witnessing an attack, accident, or other traumatic event), and, as such, our findings add to what is currently known about stress and memory by emphasizing how stress affects the initial acquisition and early consolidation of memories. Although stress at retrieval leads to pervasive disruption of memory (even, sometimes, for emotional stimuli), we have shown that stress administered prior to encoding enhances memory for emotional information, but disrupts memory for neutral information. Clearly, the specific memory process impacted by stress exposure determines to a large degree how stress influences memory.

Our findings fit well with what is known about the neurobiology of stress and memory. Both the stress-based enhance-

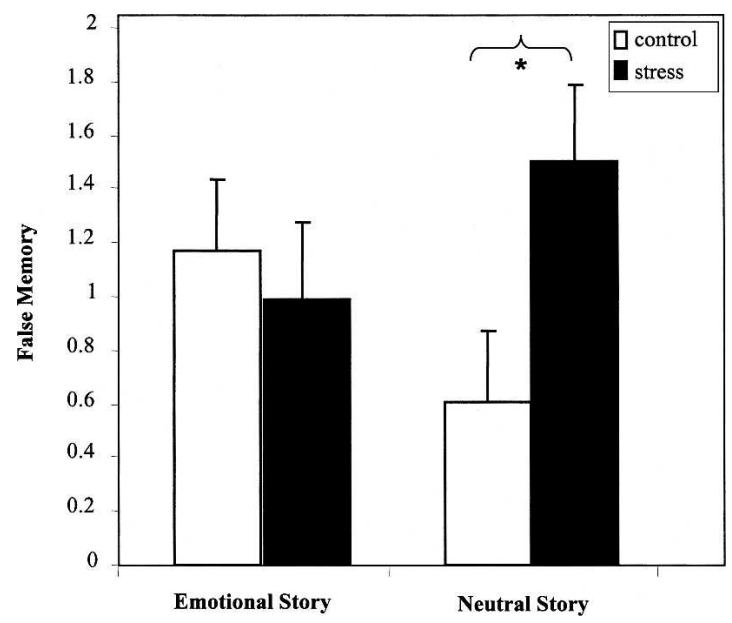

Figure 4. The impact of stress on false memory. Stressed subjects falsely recalled more slides than control subjects, but only for the neutral story.

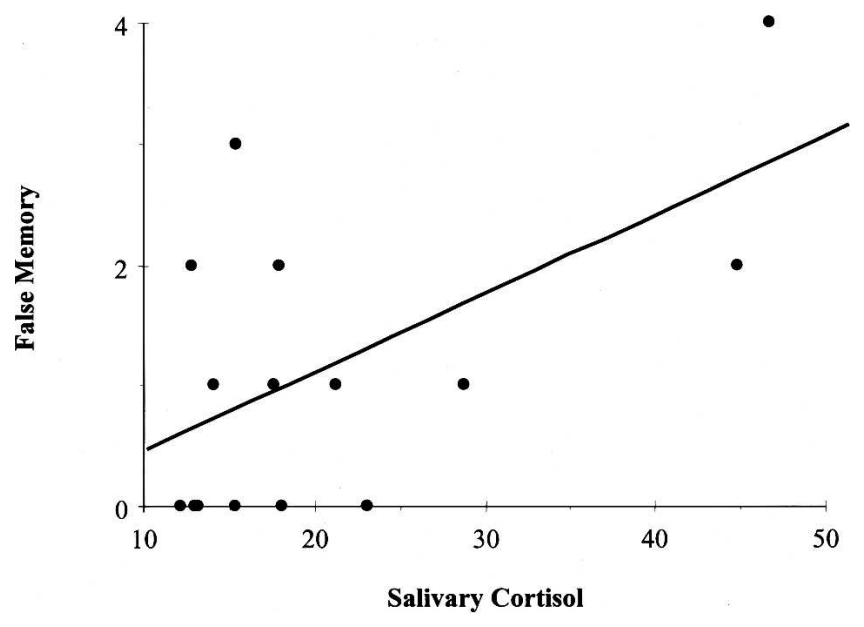

Figure 5. Positive correlation between salivary cortisol and false memory recall.

ment of emotional memory and the stress-based disruption of neutral memory likely arose from the impact of stress hormones on different memory systems. Elevations in norepinephrine and cortisol are known to facilitate the functioning of the amygdala, which is critical for emotional memory processing (Cahill and McGaugh 1998; McGaugh 2000; Kensinger and Corkin 2004; Phelps 2004). However, cortisol elevations can disrupt the functioning of the hippocampus and parts of the prefrontal cortex, which are required for normal memory function, and can result in disruptions in memory for neutral information (Kirschbaum et al. 1996; Kim and Diamond 2002; Jelicic et al. 2004; Payne et al. 2006). Through their distinct actions on these memory systems, stress hormones likely produced the divergent patterns observed for emotional and neutral memories demonstrated here.

\section{Potential mechanisms underlying stress-based emotional memory enhancement}

Animal studies have demonstrated that GC effects on emotional memory consolidation require arousal-induced increases in noradrenergic activity in the basolateral complex of the amygdala (BLA) (Roozendaal et al. 2006). Similarly, cortisol elevations in humans have been positively correlated with enhanced memory consolidation for emotionally laden information, but only in individuals who were emotionally aroused (Abercrombie et al. 2006). These findings suggest that the BLA may have been preferentially activated by our emotionally arousing slide show, thus enabling GCs to enhance memory for emotional content, but not by the neutral slide show, which neither elicited a unique arousal response nor produced further increases in noradrenergic activity. Given that cortisol levels were elevated during encoding of the arousing slides, our results provide further evidence that the arousal and stress systems must be coactivated for memory enhancement to occur (Roozendaal et al. 2001, 2006).

Considerable research has shown that interactions between the amygdala and hippocampus enhance encoding and consolidation of emotionally arousing information (Cahill 2000; Canli et al. 2000; Packard and Cahill 2001; Phelps 2004). Via these interactions, hormone release might encourage encoding and consolidation of only those memory elements that were themselves emotionally arousing and salient (Abercrombie et al. 2006; Roozendaal et al. 2006). Our results suggest that this adaptive response occurs with incredible precision-enough to differentiate between two visually identical slide shows, distinguished only by an emotionally arousing (vs. neutral) narrative. Thus, 
although hippocampal function is reduced by high cortisol levels (de Quervain et al. 2003), the hippocampus nonetheless, in its interactions with the amygdala, appears capable of processing and storing emotional memories. Because our results provide only indirect evidence that hippocampal-amygdala interactions underlie these effects, this hypothesis should be further explored using neuroimaging.

Interestingly, the hippocampus and amygdala also play interactive roles in emotional memory retrieval (Dolcos et al. 2005; Kensinger and Schacter 2005). Thus, GCs could have their deleterious impact on retrieval by feeding back to these structures and impeding normal memory retrieval operations, even while simultaneously encouraging the encoding and consolidation of new, emotionally salient memories (associated in time and space with the stressor) (Joels et al. 2006). As Roozendaal (2002) has suggested, GCs encourage a consolidation mode that is accompanied by impaired retrieval.

\section{Potential mechanisms underlying stress-based neutral memory impairment}

The neutral memory impairment we observed can also be understood in terms of the neurobiology of stress and memory. Cortisol elevations lead to demonstrable changes in hippocampal structure and function, and to pronounced memory deficits (e.g., for review, see Lupien and McEwen 1997; de Quervain et al. 2000; McEwen 2000). For example, animal studies have shown that stress exposure can lead to a suppression of synaptic and structural plasticity underlying information storage in the hippocampus (Mesches et al. 1999; Diamond et al. 2005). One recent study demonstrated that predator stress disrupted spatial memory in two groups of rats, one exposed to the stressor before training and another before memory retrieval (Diamond et al. 2006). Control rats exhibited excellent long-term memory in a water-maze task and a robust increase in the density of stubbyshaped dendritic spines in hippocampal CA1 neurons. Pretraining stress led not only to a pronounced impairment in spatial memory, but also to a reduction in hippocampal spinogenesis. Pre-retrieval stress, on the other hand, led to a similar disruption of memory, but had no effect on spinogenesis. These results suggest that specific plastic changes might underlie the long-term memory impairments frequently observed following pre-training stress exposure.

\section{Remaining questions about mechanisms}

Numerous studies suggest that amygdala activation underlies stressed-based emotional memory enhancement, whereas hippocampal inactivation underlies stress-based neutral memory impairment. However, it is not clear whether the amygdala can actually store memories, and if the hippocampus is rendered inactive by stress, how can emotional memories last long-term? It seems to us that there are two possibilities. One is that hippocampal function, while reduced under the conditions described here, is not completely inactivated nor reduced to the equivalent of a hippocampal lesion. As such, under emotional conditions, amygdala activation of the hippocampus, along with the release of memory-enhancing neuromodulators (e.g., norepinephrine), may allow at least emotional experiences (and perhaps some, but not all, aspects of neutral experiences) to be stored.

The other possibility, as specified by Diamond et al. (2007), is based on a temporal dynamics model by which hippocampal activity is briefly enhanced by stress (on the order of seconds to minutes), followed by a long refractory period during which hippocampal function is dramatically reduced. By this model, emotional and/or stressful experiences briefly intensify hippocampal processing, and this is enough time to capture the essence of an unfolding emotional episode. Shortly thereafter, however, hippocampal processing is impaired (which is why so many studies observe a deleterious effect of stress on hippocampal function). Diamond et al. (2007) can thus explain how hippocampal functioning can be impaired by stress, while also being involved in the formation of emotional memories. Interestingly, the brief activating effect of stress on hippocampal function may also explain why some neutral content remains in memory (recall that in the current experiment neutral memory was reduced compared with a control condition, but it was not lost entirely). In this brief period of activation, the hippocampus may store some small amount of neutral information-although less neutral than emotional information due to the lack of amygdala activation that allows emotional memories to be well preserved.

\section{Relevance of our findings to the false memory literature} Our findings are highly relevant to the literature on false memory and trauma. Stressed subjects falsely reported more slides from the neutral slide show than did control subjects, whereas there was no difference for the emotional slide show. This finding provides further evidence that pre-training stress exposure leads to neutral memory impairment. It also demonstrates that the improved (veridical) recall for emotional memories is not the result of a general bias shift, because there was no parallel increase in false emotional memory recall. At the same time, the impaired recall of neutral memories appears to occur despite a bias shift toward more false recall. This result parallels other findings in our laboratory. We previously showed that people are susceptible to false memories under stress (Nadel and Payne 2002; Payne et al. 2002, 2006), particularly if the false information is related to the gist or theme of a neutral episode.

\section{Caveats and study limitations}

A limitation of our study is that it did not separate stress effects at encoding from those on early consolidation processes. We accepted this limitation at the design stage of the study because we wanted to mimic what often happens in the real world, where stress precedes encoding and thus influences both encoding and early consolidation processes. Nonetheless, this decision came at a cost, as we have no way of determining when (i.e., during which memory stage) our results occurred. Previous research provides evidence for both possibilities. Payne et al. (2006) showed that stress can produce neutral memory impairment and emotional memory preservation after a brief delay, which suggests that encoding processes could support these findings. On the other hand, several pharmacological studies suggest that our results are more likely due to influences on consolidation (Buchanan and Lovallo 2001; Abercrombie et al. 2003; Kuhlmann and Wolf 2006). In these experiments, cortisol was manipulated prior to encoding, but reached its highest levels after stimulus encoding had been completed. Interestingly, Kuhlmann and Wolf (2006) demonstrated enhanced recall of emotional pictures and reduced recall of neutral pictures when cortisol was administered prior to encoding, which is very similar to the findings reported here.

There were several unexpected findings in our study that also deserve note. The first is the fact that we did not obtain generally better memory for emotional stories than the neutral ones (the effect only emerged in the stress relative to control group). This "emotional memory enhancement effect" is not only a robust finding, but one that we observed ourselves in a similar study (Payne et al. 2006). That study was similar to the one presented here, except that Payne et al. (2006) examined memory for a single episode that contained both neutral and emotional components. In that study, memory for the emotional 
content was clearly superior to memory for neutral content in both the stress and control groups. One possible explanation for our failure to observe that effect here is the presence of emotional content in both the emotional and neutral slide shows (recall that both slide shows contain the same emotional visual content and are differentiated only by the narrative), which could make both slide shows emotional enough to prevent a difference from emerging in the control group. This explanation is lacking, however, because Cahill and colleagues (e.g., Cahill et al. 1994) often see the emotional memory enhancement effect in their control groups.

The second unanticipated finding concerns the lack of sex differences in the memory analyses. This may have been due to our focus on encoding and early consolidation processes (but, see Payne et al. 2006 and Payne et al. 2002, the former of which did and the latter of which did not find sex differences when stress was administered before encoding), to the timing of the menstrual cycle in the females in our study (which we did not record), or perhaps to some other unknown factor. Why these effects emerge in some, but not all, studies is currently unknown. Nonetheless, we note that this finding is at odds with many other studies of stress and memory, including some of our own (e.g., Payne et al. 2006) (for review, see Wolf et al. 2001). Future studies could improve upon the current design in several ways. Most importantly, they should carefully test the impact of stress on encoding, consolidation, and retrieval separately, across different types of memory (e.g., emotionally arousing negative and positive, neutral), different memory paradigms (e.g., verbal, pictoral), and different delay intervals, while carefully controlling for time of day and menstrual cycle in female participants.

\section{Concluding comments}

To date, most studies of human stress and memory have focused either on the impairing effect of stress on retrieval, or the (generally) enhancing effect of stress on consolidation. We add to these findings by demonstrating that stress administered prior to encoding can influence later remembering, and that these effects are quite complex. We have shown that the influence of stress hormones administered prior to learning is different from that of hormones administered before retrieval. Given that many of the legal and clinical issues surrounding stress and memory arise from situations where individuals encode information under stress and then try to recall it at some point in the future, this finding seems particularly relevant (Payne et al. 2004).

In summary, we have demonstrated that stress administered before encoding can enhance memory for an emotional event but disrupt memory for a carefully matched neutral event. These findings suggest that the interaction between stress hormones and arousal influences which events should be preferentially remembered and which should be forgotten.

\section{Materials and Methods}

Seventy-six University of Arizona undergraduate students (44 females, 32 males) were tested in two experimental sessions, $1 \mathrm{wk}$ apart. In a 2 (stress vs. control) $\times 2$ (neutral memory vs. emotional memory) between-subjects factorial design; 19 participants were assigned to the stress-emotional memory condition, 17 to the stress-neutral memory condition, 20 to the controlemotional memory condition, and 20 to the control-neutral memory condition. Eleven subjects failed to return at the 1-wk follow up session, resulting in a total of 65 subjects who completed the entire protocol.

In the first experimental session, participants arrived at the lab, gave informed consent, and were told their physiological responses would be monitored during a speech and throughout the first part of the experiment. We were thus able to collect heart-rate data (see below) while simultaneously persuading par- ticipants that the experiment was an investigation of "physiological sensitivity to stimuli" rather than memory. Participants were then exposed to the Trier Social Stress Test (TSST) (Kirschbaum et al. 1993), or a control treatment, both of which lasted $\sim 20$ min. Immediately after the TSST or control treatment, participants viewed either an emotionally arousing or a matched emotionally neutral slide show. One week later, they returned for a surprise memory test.

\section{Stress and control treatments}

The TSST is a well-established method used to induce a significant HPA response in laboratory settings (Kirschbaum et al. 1993, 1996; Kuhlmann et al. 2005b). The TSST combines social evaluative threat with stressor uncontrollability, which together produce a large HPA response in humans (Dickerson and Kemeny 2004). The TSST consists of a 10-min speech preparation period, followed by a 5 -min speech given without notes (notes are abruptly taken away from participants prior to their speech).

Participants delivered their speeches in the presence of individuals wearing white lab coats and were given the impression that their performance was being audio and video recorded for later analysis. Immediately following the speech, participants performed an arithmetic task aloud (count backward from 1022 by 13 's as quickly as possible for $5 \mathrm{~min}$ ). Upon making a mistake, they were told, "No, that is not correct. Start over with $1022 . "$ Participants in the control condition perused magazines containing trivial content for $\sim 20 \mathrm{~min}$. They were monitored to ensure that they followed these directions and remained awake.

\section{Stimulus materials}

Memory was tested using a procedure developed by Heuer and Reisberg (1990) and Cahill et al. (1994) to investigate memory for closely matched neutral and emotional episodes depicted in a slide show (Cahill and McGaugh 1995). After completing the TSST or control procedure, participants were asked to carefully attend to each of 11 color slides while listening to either a neutral or emotional narrative (Cahill et al. 1994; Cahill and McGaugh 1995). They were instructed to view each slide for the duration of its presentation, and were told that they might find some slides pleasant, some unpleasant, and some neutral. Each slide was presented for 6 sec on a computer monitor and was accompanied by a recorded narration presented over headphones.

The slide show depicts a car accident and resulting emergency surgery. In the emotional version, the narration describes an event in which a car struck a boy who was subsequently taken to a hospital for surgery. In the neutral version, the narration described these same events as a mock hospital "disaster drill."

The slide show contains three distinct phases. Phases 1 and 3 are neutral in both images and narration, and they are identical in both conditions. The emotional narration is introduced in Phase 2 (i.e., during presentation of slides 5-7, which depict the accident and surgery) of the emotional condition only.

\section{Memory assessment}

Participants returned $1 \mathrm{wk}$ later for an unexpected memory test, which consisted of three parts: free recall, cued recall, and recognition tests. First, free recall was assessed by asking participants to write down everything they could remember about the slide show they had seen the previous week (Heuer and Reisberg 1990; Cahill et al. 1994). Their performance was compared with a comprehensive list of 125 elements of information about the story contained in the slides and narration, such as "the father worked at the hospital" and "the boy had blonde hair." Participants were credited with recalling a story element if it was explicitly mentioned in their responses. Two judges, blind to experimental condition, scored these data. Percent agreement between the judges was $>90 \%$.

Cued recall was then assessed by reminding participants that they had seen a total of 11 slides, and asking them to recall each slide in as much detail as possible. Participants were credited with remembering a slide if they recalled information that could only be known from having viewed that particular slide (Cahill et 
al. 1994). Two judges, blind to experimental condition, scored these data. If a participant's cued-recall response included an ambiguous or vague description, like "there was a slide with a car," the free-recall response was consulted for verification. Reports of slides that were clearly not part of the slide show (e.g., "a slide showing an ambulance") were scored as a false slide report. These false reports were of special interest given our previous finding that stress increases false memories (Payne et al. 2002).

Finally, participants received a four-alternative forced choice recognition questionnaire that included eight questions per slide (88 questions total), assessing memory for both the general theme and details of the slide show. The questions and answer choices were presented on a computer screen and participants responded using a keyboard.

\section{Measures of stress responsivity}

\section{Cortisol}

Cortisol was assessed via saliva, which provides an index of bioavailable free cortisol and is considered a more accurate measure of cortisol responsivity than plasma cortisol (Kudielka and Kirschbaum 2005). Saliva samples were collected with Salivette collection devices (Sarstedt, Inc.), and were taken: (1) at the beginning of the experiment, before the stress manipulation, (2) immediately after the stress manipulation, (3) $30 \mathrm{~min}$ following the manipulation, and (4) at the beginning of the second visit, 1 wk later (Fig. 6). On each visit, participants rinsed their mouths 10 min before the first saliva sample.

Using in-house facilities, all saliva samples were assayed for cortisol in duplicate with a commercially available enzyme immunoassay (Salimetrics, LLC). The test used $25 \mu \mathrm{L}$ of saliva per determination, had a lower limit of sensitivity of $0.003 \mu \mathrm{g} / \mathrm{dl}$, standard curve range of from 0.007 to $1.8 \mu \mathrm{g} / \mathrm{dl}$, and average intra- and interassay coefficients of variation $5.1 \%$ and $8.2 \%$, respectively. Method accuracy, determined by spike recovery, and linearity, determined by serial dilution were $103 \%$ and $96 \%$, respectively.

\section{Catecholamines}

$\alpha$-Amylase is an enzyme secreted from salivary glands in response to sympathetic activation (Schneyer and Hall 1991). Stressrelated increases in salivary $\alpha$-amylase can be inhibited by adrenergic antagonists and can be stimulated by $\beta$-adrenergic agonists (Speirs et al. 1974; Gallacher and Petersen 1983). Correspondingly, in response to psychological stress, salivary $\alpha$-amylase is a correlate of noradrenergic and adrenergic release, but not cortisol release (Chatterton Jr. et al. 1996; Nater et al. 2005). Given these findings, salivary $\alpha$-amylase (pre- and post-stress manipulation) was included in this study as an exploratory surrogate marker of catecholamine response to stress, a stress response system distinct from the cortisol system.

A testing service (Salimetrics, LLC) assayed the saliva samples collected before and after the experimental manipulation for $\alpha$-amylase in singlet with an enzyme immunoassay. The test used $25 \mu \mathrm{L}$ of saliva per determination and had average intraand interassay coefficients of variation $5.5 \%$ and $4.7 \%$, respec-

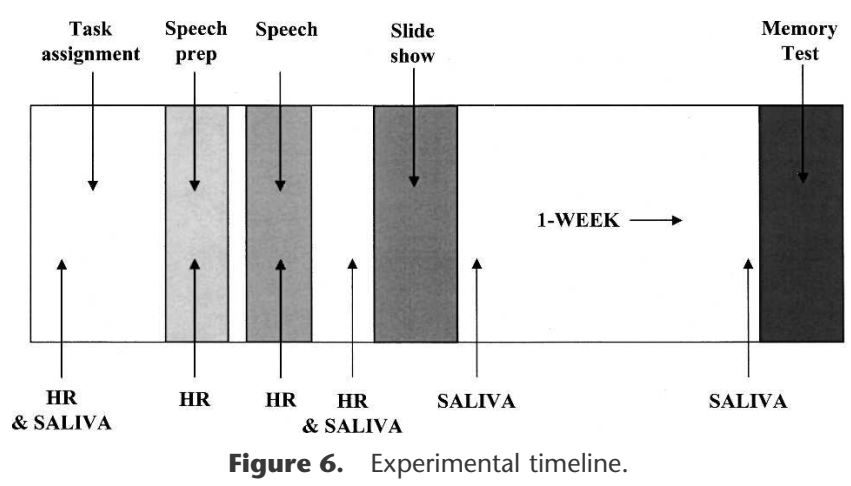

tively. Method accuracy, determined by spike recovery, and linearity, determined by serial dilution were $101 \%$ and $96 \%$, respectively.

\section{Heart rate}

An ECG amplifier attached to three leads with disposable snap electrodes (Biopac) acquired electrocardiogram (ECG) signals. The experimenter cleaned skin on the abdomen and left and right lower ribs with abrasive pads soaked in rubbing alcohol. Two electrodes were affixed to the ribs, while the ground electrode was affixed to the abdomen. Mean heart rate (HR) was calculated over each of four 2-min intervals: (1) before participants were notified of their group assignment, (2) during preparation for the speech task TSST (or during the rest period for the control subjects), (3) during the speech task for the stress participants (or at the same clock time for control subjects), and (4) after completing the stress or control procedure (Fig. 6).

\section{Subjective stress ratings}

Subjective anxiety reports, using the Spielberger State Anxiety Inventory (Spielberger et al. 2004), were collected at four time points: (1) at the beginning of the experiment, before the experimental manipulation, (2) immediately after the manipulation, (3) immediately after the second anxiety measure (participants completed a modified version of the inventory asking them, "How did you feel during the speech?"), and (4) at the beginning of the second visit, $1 \mathrm{wk}$ later (Fig. 6).

\section{Acknowledgments}

We thank Linda Jarrell and Marie Osbourne for their assistance with subject running. We also thank Elizabeth Kensinger, Jill Lany, Dan Payne, Sam Payne, Dan Schacter, and Bob Stickgold for their helpful comments on the manuscript.

\section{References}

Abercrombie, H.C., Kalin, N.H., Thurow, M.E., Rosenkranz, M.A., and Davidson, R.J. 2003. Cortisol variation in humans affects memory for emotionally laden and neutral information. Behav. Neurosci. 117: $505-516$

Abercrombie, H.C., Speck, N.S., and Monticelli, R.M. 2006. Endogenous cortisol elevations are related to memory facilitation only in individuals who are emotionally aroused. Psychoneuroendocrinology 31: $187-196$.

Buchanan, T.W. and Lovallo, W.R. 2001. Enhanced memory for emotional material following stress-level cortisol treatment in humans. Psychoneuroendocrinology 26: 307-317.

Buchanan, T.W., Tranel, D., and Adolphs, R. 2006. Impaired memory retrieval correlates with individual differences in cortisol response but not autonomic response. Learn. Mem. 13: 382-387.

Cahill, L. 2000. Neurobiological mechanisms of emotionally influenced, long-term memory. Prog. Brain Res. 126: 29-37.

Cahill, L. and McGaugh, J.L. 1995. A novel demonstration of enhanced memory associated with emotional arousal. Conscious. Cogn. 4: $410-421$.

Cahill, L. and McGaugh, J.L. 1998. Mechanisms of emotional arousal and lasting declarative memory. Trends Neurosci. 21: 294-299.

Cahill, L., Prins, B., Weber, M., and McGaugh, J.L. 1994. $\beta$-adrenergic activation and memory for emotional events. Nature 371: 702-704.

Cahill, L., Gorski, L., and Le, K. 2003. Enhanced human memory consolidation with post-learning stress: Interaction with the degree of arousal at encoding. Learn. Mem. 10: 270-274.

Canli, T., Zhao, Z., Brewer, J., Gabrieli, J.D., and Cahill, L. 2000. Event-related activation in the human amygdala associates with later memory for individual emotional experience. J. Neurosci. 20: RC99.

Chatterton Jr., R.T., Vogelsong, K.M., Lu, Y.C., Ellman, A.B., and Hudgens, G.A. 1996. Salivary $\alpha$-amylase as a measure of endogenous adrenergic activity. Clin. Physiol. 16: 433-448.

de Quervain, D.J., Roozendaal, B., Nitsch, R.M., McGaugh, J.L., and Hock, C. 2000. Acute cortisone administration impairs retrieval of long-term declarative memory in humans. Nat. Neurosci. 3: 313-314.

de Quervain, D.J., Henke, K., Aerni, A., Treyer, V., McGaugh, J.L., Berthold, T., Nitsch, R.M., Buck, A., Roozendaal, B., and Hock, C. 2003. Glucocorticoid-induced impairment of declarative memory retrieval is associated with reduced blood flow in the medial temporal lobe. Eur. J. Neurosci. 17: 1296-1302. 
Diamond, D.M., Park, C.R., Campbell, A.M., and Woodson, J.C. 2005. Competitive interactions between endogenous LTD, LTP in the hippocampus underlie the storage of emotional memories and stress-induced amnesia. Hippocampus 15: 1006-1025.

Diamond, D.M., Campbell, A.M., Park, C.R., Woodson, J.C., Conrad C.D., Bachstetter, A.D., and Mervis, R. 2006. Influence of predator stress on the consolidation versus retrieval of long-term spatial memory and hippocampal spinogenesis. Hippocampus 16: 571-576.

Diamond, D.M., Campbell, A.M., Park, C.R., Halonen, J., and Zoladz, P.R. 2007. The temporal dynamics model of emotional memory processing: A synthesis on the neurobiological basis of stress-induced amnesia, flashbulb and traumatic memories, and the Yerkes-Dodson Law. Neural Plast. 2007: 60803.

Dickerson, S.S. and Kemeny, M.E. 2004. Acute stressors and cortisol responses: A theoretical integration and synthesis of laboratory research. Psychol. Bull. 130: 355-391.

Dolcos, F., LaBar, K.S., and Cabeza, R. 2005. Remembering one year later: Role of the amygdala and the medial temporal lobe memory system in retrieving emotional memories. Proc. Natl. Acad. Sci. 102: $2626-2631$.

Elzinga, B.M. and Roelofs, K. 2005. Cortisol-induced impairments of working memory require acute sympathetic activation. Behav. Neurosci. 119: 98-103.

Gallacher, D.V. and Petersen, O.H. 1983. Stimulus-secretion coupling in mammalian salivary glands. Int. Rev. Physiol. 28: 1-52.

Heuer, F. and Reisberg, D. 1990. Vivid memories of emotional events: The accuracy of remembered minutiae. Mem. Cognit. 18: 496-506.

Jelicic, M., Geraerts, E., Merckelbach, H., and Guerrieri, R. 2004. Acute stress enhances memory for emotional words, but impairs memory for neutral words. Int. J. Neurosci. 114: 1343-1351.

Joels, M., Pu, Z., Wiegert, O., Oitzl, M.S., and Krugers, H.J. 2006. Learning under stress: How does it work? Trends Cogn. Sci. 10: $152-158$.

Kensinger, E.A. and Corkin, S. 2004. Two routes to emotional memory: Distinct neural processes for valence and arousal. Proc. Natl. Acad. Sci. 101: 3310-3315.

Kensinger, E.A. and Schacter, D.L. 2005. Retrieving accurate and distorted memories: Neuroimaging evidence for effects of emotion. Neuroimage 27: 167-177.

Kim, J.J. and Diamond, D.M. 2002. The stressed hippocampus, synaptic plasticity and lost memories. Nat. Rev. Neurosci. 3: 453-462.

Kirschbaum, C., Pirke, K.M., and Hellhammer, D.H. 1993. The "Trier Social Stress Test"-A tool for investigating psychobiological stress responses in a laboratory setting. Neuropsychobiology 28: 76-81.

Kirschbaum, C., Wolf, O.T., May, M., Wippich, W., and Hellhammer, D.H. 1996. Stress- and treatment-induced elevations of cortisol levels associated with impaired declarative memory in healthy adults. Life Sci. 58: 1475-1483.

Kudielka, B.M. and Kirschbaum, C. 2005. Sex differences in HPA axis responses to stress: A review. Biol. Psychol. 69: 113-132.

Kuhlmann, S. and Wolf, O.T. 2006. Arousal and cortisol interact in modulating memory consolidation in healthy young men. Behav. Neurosci. 120: 217-223.

Kuhlmann, S., Kirschbaum, C., and Wolf, O.T. 2005a. Effects of oral cortisol treatment in healthy young women on memory retrieval of negative and neutral words. Neurobiol. Learn. Mem. 83: 158-162.

Kuhlmann, S., Piel, M., and Wolf, O.T. 2005b. Impaired memory retrieval after psychosocial stress in healthy young men. J. Neurosci. 25: 2977-2982.

Lupien, S.J. and McEwen, B.S. 1997. The acute effects of corticosteroids on cognition: Integration of animal and human model studies. Brain Res. Brain Res. Rev. 24: 1-27.

Lupien, S.J. and Lepage, M. 2001. Stress, memory, and the hippocampus: Can't live with it, can't live without it. Behav. Brain Res. 127: 137-158.

McEwen, B.S. 2000. The neurobiology of stress: From serendipity to clinical relevance. Brain Res. 886: 172-189.

McGaugh, J.L. 2000. Memory-a century of consolidation. Science 287: 248-251.
Mesches, M.H., Fleshner, M., Heman, K.L., Rose, G.M., and Diamond, D.M. 1999. Exposing rats to a predator blocks primed burst potentiation in the hippocampus in vitro. J. Neurosci. 19: RC18.

Nadel, L. and Jacobs, W.J. 1998. Traumatic memory is special. Curr. Dir. Psychol. Sci. 7: 154-157.

Nadel, L. and Payne, J.D. 2002. The relationship between episodic memory and context: Clues from memory errors made while under stress. Physiol. Res. (Suppl 1) 51: S3-S11.

Nater, U.M., Rohleder, N., Gaab, J., Berger, S., Jud, A., Kirschbaum, C., and Ehlert, U. 2005. Human salivary $\alpha$-amylase reactivity in a psychosocial stress paradigm. Int. J. Psychophysiol. 55: 333-342.

Packard, M.G. and Cahill, L. 2001. Affective modulation of multiple memory systems. Curr. Opin. Neurobiol. 11: 752-756.

Payne, J.D., Nadel, L., Allen, J.J., Thomas, K.G., and Jacobs, W.J. 2002. The effects of experimentally induced stress on false recognition. Memory 10: $1-6$.

Payne, J.D., Nadel, L., Britton, W.B., and Jacobs, W.J. 2004. The biopsychology of trauma and memory. In Memory and emotion. (eds. D. Reisberg and P. Hertel), pp. 76-128. Oxford University Press, UK.

Payne, J.D., Jackson, E.D., Ryan, L., Hoscheidt, S., Jacobs, J.W., and Nadel, L. 2006. The impact of stress on neutral and emotional aspects of episodic memory. Memory 14: 1-16.

Phelps, E.A. 2004. Human emotion and memory: Interactions of the amygdala and hippocampal complex. Curr. Opin. Neurobiol. 14: 198-202.

Putman, P., Van Honk, J., Kessels, R.P., Mulder, M., and Koppeschaar, H.P. 2004. Salivary cortisol and short and long-term memory for emotional faces in healthy young women. Psychoneuroendocrinology 29: 953-960.

Roozendaal, B. 2002. Stress and memory: Opposing effects of glucocorticoids on memory consolidation and memory retrieval. Neurobiol. Learn. Mem. 78: 578-595.

Roozendaal, B., de Quervain, D.J., Ferry, B., Setlow, B., and McGaugh, J.L. 2001. Basolateral amygdala-nucleus accumbens interactions in mediating glucocorticoid enhancement of memory consolidation. J. Neurosci. 21: 2518-2525.

Roozendaal, B., Okuda, S., de Quervain, D.J., and McGaugh, J.L. 2006. Glucocorticoids interact with emotion-induced noradrenergic activation in influencing different memory functions. Neuroscience 138: 901-910.

Schneyer, C.A. and Hall, H.D. 1991. Effects of varying frequency of sympathetic stimulation on chloride and amylase levels of saliva elicited from rat parotid gland with electrical stimulation of both autonomic nerves. Proc. Soc. Exp. Biol. Med. 196: 333-337.

Speirs, R.L., Herring, J., Cooper, W.D., Hardy, C.C., and Hind, C.R. 1974. The influence of sympathetic activity and isoprenaline on the secretion of amylase from the human parotid gland. Arch. Oral Biol. 19: $747-752$.

Spielberger, C.D., Reheiser, E.C., Hilsenroth, M.J., and Segal, D.L. 2004 Measuring anxiety, anger, depression, and curiosity as emotional states and personality traits with the STAI, STAXI and STPI. In Comprehensive handbook of psychological assessment, Vol. 2: Personality assessment. pp. 70-86. John Wiley \& Sons, Hoboken, NJ.

Tops, M., van der Pompe, G., Baas, D., Mulder, L.J., Den Boer, J.A., Meijman, T.F., Korf, J. 2003. Acute cortisol effects on immediate free recall and recognition of nouns depend on stimulus valence. Psychophysiology 40: 167-173.

Vyas, A., Mitra, R., Rao, S.B.S., and Chatterji, S. 2002. Chronic stress induces contrasting patterns of dendritic remodeling in hippocampal and amygdaloid neurons. J. Neurosci. 22: 6810-6818.

Wolf, O.T., Schommer, N.C., Hellhammer, D.H., McEwen, B.S., and Kirschbaum, C. 2001. The relationship between stress induced cortisol levels and memory differs between men and women. Psychoneuroendocrinology 26: 711-720.

Received August 16, 2007; accepted in revised form October 4, 2007. 


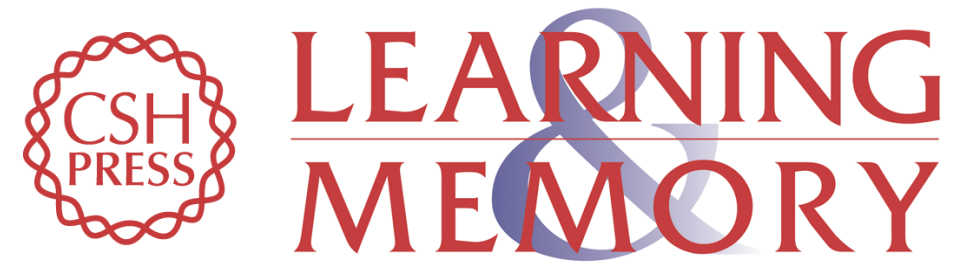

\section{Stress administered prior to encoding impairs neutral but enhances emotional long-term episodic memories}

Jessica D. Payne, Eric D. Jackson, Siobhan Hoscheidt, et al.

Learn. Mem. 2007, 14:

Access the most recent version at doi:10.1101//m.743507

References This article cites 53 articles, 10 of which can be accessed free at:

http://learnmem.cshlp.org/content/14/12/861.full.html\#ref-list-1

License

Email Alerting

Receive free email alerts when new articles cite this article - sign up in the box at the Service top right corner of the article or click here. 